\title{
A study on the effect of electrical stimulation as a user stimuli for motor imagery classification in Brain-Machine Interface
}

\author{
Saugat Bhattacharyya (1), Maureen Clerc (2), Mitsuhiro Hayashibe (1) \\ (1) BCI-LIFT project, CAMIN Team, INRIA-LIRMM, University of Montpellier, France; (2) \\ BCI-LIFT project, Athena Team, Inria Sophia Antipolis, France.
}

This article is distributed under the terms of the Creative Commons Attribution Noncommercial License (CC BY-NC 4.0) which permits any noncommercial use, distribution, and reproduction in any medium, provided the original author(s) and source are credited.

\begin{abstract}
Functional Electrical Stimulation (FES) provides a neuroprosthetic interface to non-recovered muscle groups by stimulating the affected region of the human body. FES in combination with Brain-machine interfacing (BMI) has a wide scope in rehabilitation because this system directly links the cerebral motor intention of the users with its corresponding peripheral muscle activations. In this paper, we examine the effect of FES on the electroencephalography (EEG) during motor imagery (left- and right-hand movement) training of the users. Results suggest a significant improvement in the classification accuracy when the subject was induced with FES stimuli as compared to the standard visual one.
\end{abstract}

Key Words: Electrical Stimulation, Brain machine interfacing, Motor Imagery, Electroencephalography

The advent of Brain-Machine Interfacing (BMI) has opened a direct communication pathway between the brain and the environment in the form of robots, prosthesis, wheelchair or a computer without any muscle intervention. ${ }^{1}$ BMI has immense potential in facilitating rehabilitation for patients suffering from stroke, amyotrophic lateral sclerosis, spinal injury and physical disability1. A BMI system would drive a neuroprosthetic device to bridge the gap between the central nervous system and peripheric muscles and in some instance even a separate prosthesis. Electroencephalography (EEG) signals is the most commonly used BMI recording device and signals related to limb movement (known as motor imagery signals) are elicited as Event Related Desynchronization/Synchronization (ERD/ERS) response ${ }^{2,3}$. The BMI system decodes the ERD/ERS signals to determine the movement as intended by the user. Functional Electrical Stimulation (FES) ${ }^{4,5}$ aims at directly stimulating the muscles on the affected side of the patient and it is reported that FES is capable of reconstructing certain daily life skills6. Till date, many researchers $^{7-9}$ have combined BMI with FES to directly link the mental intention of the user with a muscular response. In such a system, the BMI detects the motor intentions of the user which in turn activates the FES device. FES activation may affect the sensory pathways, if intact, which may, in turn impact the cortical activity. Such phenomena may influence the performance of the BMI. Gollee et al. ${ }^{8}$ found no major influence of FES on
Steady-State Visually Evoked Potential (SSVEP) signal. Takahashi et al. ${ }^{9}$ also found no effect of FES on leg stimulation. In this paper, we report the preliminary results of the effect of electrical stimulation during a motor imagery training task on healthy subjects and its comparison with visual stimuli. Through this study, we aim at employing FES as a proprioceptive feedback to the brain to improve the learning of the subject both in terms of accuracy and time. If successful, this system can accelerate the motor recovery process of the patient by enhancing the motor learning in the brain.

\section{Materials and Methods}

The EEG recording is conducted using a 14 channel Emotiv Epoc neuro-headset with a sampling rate of $128 \mathrm{~Hz}$ and an in-built band-pass filter of $0.2-45 \mathrm{~Hz}$. The electrodes: AF3, F7, F3, FC5, T7, P7, O1, O2, P8, T8, FC6, F4, F8 and AF4, are arranged on the basis of the standard 10-20 system10. Three healthy male subjects (2 right handed and one left handed), participated in this experiment. Two subjects participated in the BMI experiment as a first experience whereas one subject was an experienced BMI user. In this experiment, we abide by the norms of Helsinki Declaration of 1975, revised in 2000. Prior to the experiments, the subjects are informed about the purpose of the experiment and the tasks they have to perform. Surface electrical stimulation is transmitted to induce the hand extension tilting towards the back of 


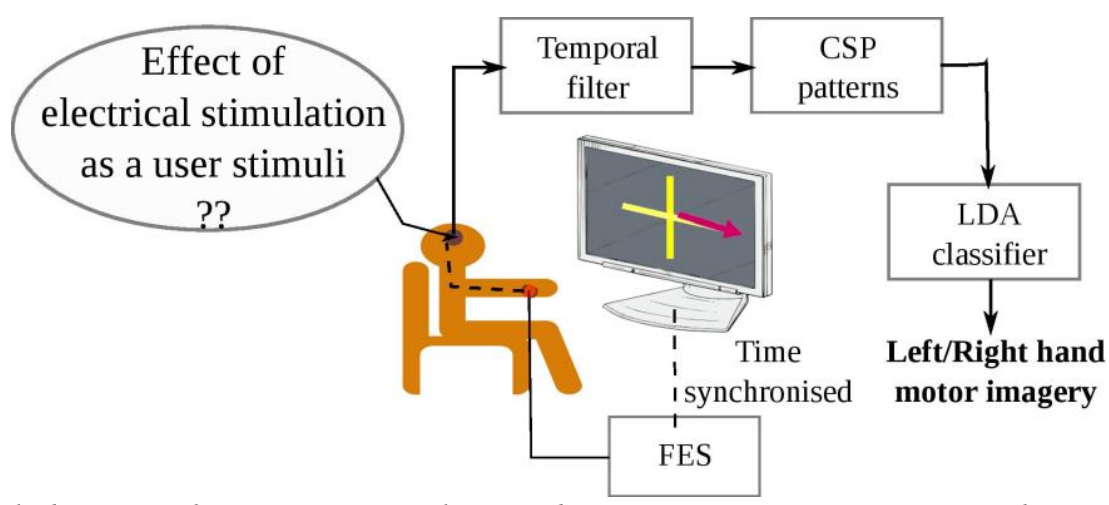

Fig 1. Block diagram of our experimental setup during motor imagery sessions where user stimuli is with conventional visual stimuli, electrical stimulation stimuli or the combined, respectively

the hand by applying the stimulation to the extensor digitorum muscle. It is applied to each side of the hand respectively, corresponding to right/left motor imagery task. The stimulus is delivered by a computer-controlled stimulator (ProStim, MXM, France) with PW modulation ( $\mathrm{PW} \max =450$ us) at a constant amplitude and frequency $(30 \mathrm{~Hz})$. Each stimulation sequence consists of a trapezoidal envelope train of PW $(0.4 \mathrm{~s}$ ramp-up, $1.2 \mathrm{~s}$ plateau, $0.4 \mathrm{~s}$ ramp-down). The experiment designed for this work is divided into three sessions: only visual, only FES and both visual-FES stimuli. The sessions consist of instructing the subjects through a sequence of repetitive stimuli to execute the corresponding motor imagery task, which in our case, is left and right hand movement. One sequence of stimulus is known as trial. A trial for the visual stimulus is designed as follows: First, a blank screen is projected to the subject for $20 \mathrm{~s}$, which provides the baseline of the EEG. Then, a fixation ' + ' is displayed on the screen for $1 \mathrm{~s}$ which is an indicator to the subject to get ready for the task. Next, the instructions are provided to the subject for 3 seconds in the form of arrows. According to the direction of the arrow, the subject imagines either left or right hand movement. Following the instructions, a blank screen is again displayed for $1.5 \mathrm{~s}-3.5 \mathrm{~s}$. The FES session is similar to the visual one except in place of the arrows, stimulation is directly induced in the fore-arm of the hand of interest, without providing any visual information. In the Visual-FES session, both the combined stimulations are time-synchronized to each other. Each session is composed of 40 trials (20 for each movement). After acquisition, the incoming raw EEG signal is band-pass filtered at 8-30 Hz. Then, common spatial filters (CSP) is applied to extract features relevant to left- and right-motor hand movement EEG signals. CSP is a spatial filter widely used in BMI because the spatial patterns contain highly discriminative features between two classes. ${ }^{11}$ In this study, we prepare the feature vectors using 6 spatial filters which is then transferred as inputs to a linear discriminant analysis (LDA) classifier. ${ }^{12}$ Finally, the classifier detects the corresponding motor intention of the subject, i.e., left and right motor movement. A block diagram of our experimental setup during Visual-FES session is illustrated in Fig. 1.

\section{Results}

In this paper, we first attempt to observe the difference of the spatial patterns during the sessions: visual, FES and visual+FES. For this purpose, we determine the power spectral density (PSD)13 of each spatial filters. The PSD distribution for six spatial filters of subject 1 is shown in Fig. 2. As observed from the figure, the patterns are highly discriminable for the first four filters and they overlap in the last two. it is also noted that FES+Visual session (in black) has the highest power followed by Visual (in blue) session and lastly FES session (in red) for the first three filters. A similar trend is noted for the other two subjects too. Next, we employ 10 cross validation 12 on the dataset, where at every iteration, one block of data is randomly selected for testing and the rest of the block are employed for training. Table I presents the average classification accuracy (over 10 runs) and it is noted that for Subject 01 and 02 (with first BMI experience) the accuracy during FES session increases by $6.28 \%$ and $5.51 \%$ as compared its visual counterpart. The difference between the FES and Visual+FES sessions are small. On the other hand, Subject 03 (with previous BMI experience) has a better result during the visual session than the FES session. Thus, it can be inferred from this result that subjects with no prior BMI training of motor imagery show a significant improvement in learning when induced with FES.

\section{Discussion}

In this paper, we aimed at studying the effect of electrical stimulation as a user stimuli for motor imagery classification (left and right hand movement) in BMI. Fig. 2 shows a significant difference of the spatial patterns during the three stimuli sessions: visual, FES and visual+FES. Results on Table I also shows a significant rise in accuracy for 2 (of 3) subjects which suggest a positive influence of FES during motor imagery training of the subjects. It was noted that both the subjects had no previous experience 


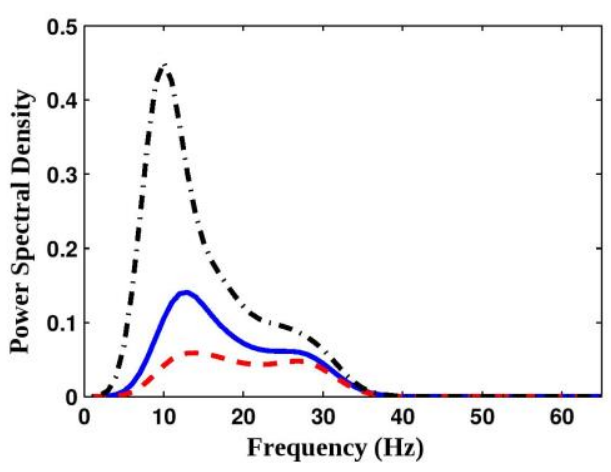

(a) Spatial Filter 1

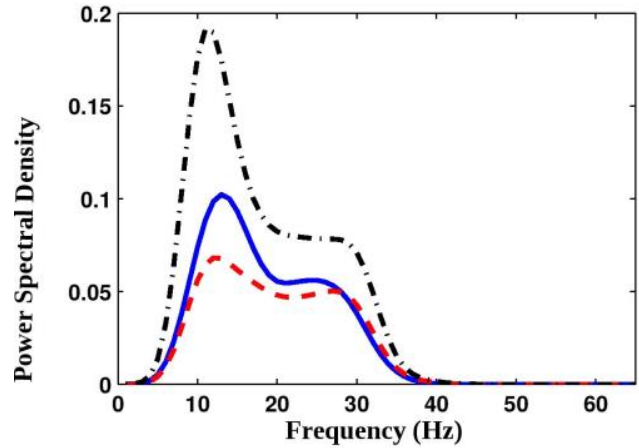

(c) Spatial Filter 3

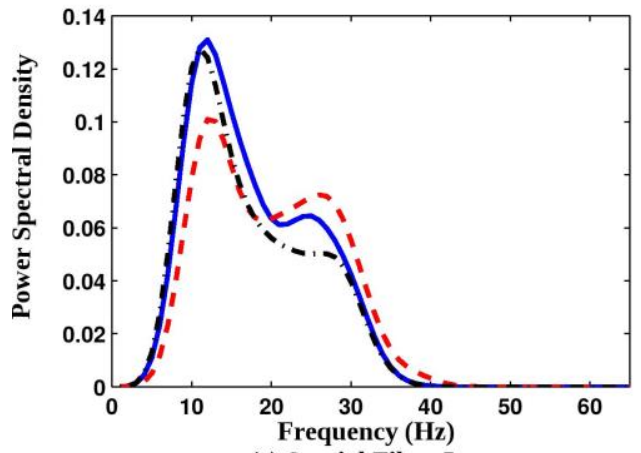

(e) Spatial Filter 5

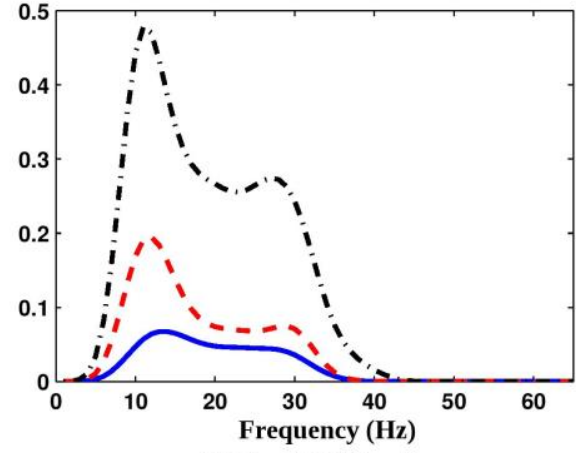

(b) Spatial Filter 2

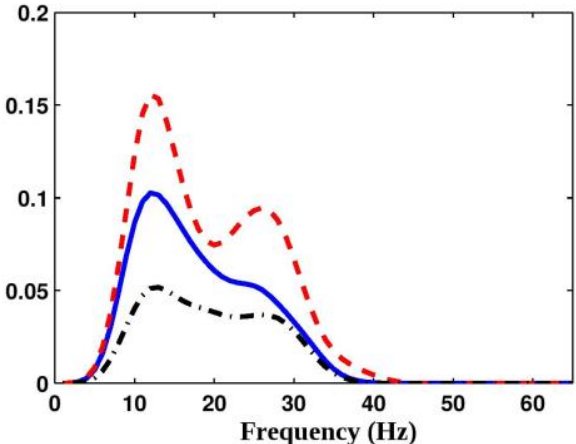

(d) Spatial Filter 4

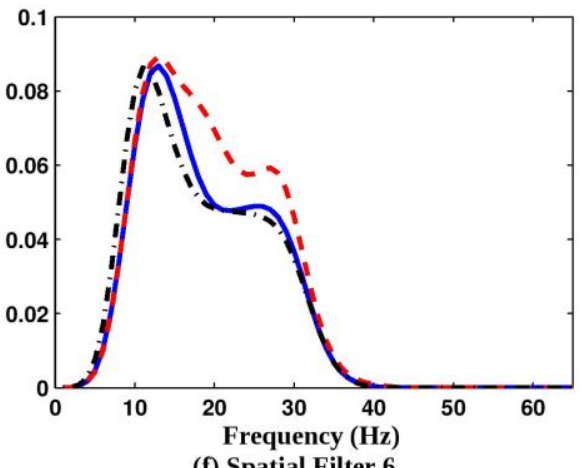

(f) Spatial Filter 6

Fig 2. Power spectral density distribution of the six filters during Visual (in blue-), FES (in red --) and Visual+FES (in black -.) sessions.

on BMI, then they were not familiar with generating motor imagery with visual stimuli. Visual stimuli are the widely accepted form of motor training but the subject requires constant training to reach an optimal result. Based on the results of this study, we can infer that electrical stimulation can also be used for motor training and it can potentially provide better performance as it can make natural proprioceptive feedback related to motor performance than visual stimuli which requires user's recognition regarding the visual cue. Further studies on a larger group of subjects are required to validate this claim. Future studies in this research will include studying the effect of FES as proprioceptive neuro-feedback training to BMI. This will lead to an improvement in motor imagery classification which helps would aid in neuro-prosthetic or robot control ${ }^{14}$.

\section{Contributions}

Saugat Bhattacharyya: Experiment preparation, data acquisition and processing, publication writing

Maureen Clerc: Experiment preparation, Data acquisition, supervision of data processing and publication drafting, publication review.

Mitsuhiro Hayashibe: Experiment preparation, Data acquisition, supervision of data processing and publication drafting, publication review.

\section{Acknowledgement}

The authors would like to thank the participants in this study. The paper is presented at IFESS Conference 2016.

\section{Conflict of Interest}


The author declare no potential conflict of interests.

\section{Corresponding Author}

Saugat Bhattacharyya, LIRMM, Université de Montpellier, Campus Saint Priest - Bat 5 - Bureau 03/248, 860 Rue de St Priest, 34095 Montpellier cedex 5 France. E-mail: saugat.bhattacharyya@inria.fr

E-mails of coAuthors

Maureen Clerc: Maureen.clerc@inria.fr

Mitsuhiro Hayashibe: Mitsuhiro;hayashibe@inria.fr

\section{References}

1. Dornhege G, Millan JdR, Hinterberger T, McFarland DJ, Muller K-R, eds. Toward Brain-computer Interfacing, ser. A Bradford book. MIT Press; 2007.

2. Bhattacharyya S, Konar A Tibarewala DN. Motor imagery, P300 and error-related eeg-based robot arm movement control for rehabilitation purpose. Med \& Biological Eng \& Comp 2014;52:1007-17.

3. Thomas E, Dyson M, Clerc M. An analysis of performance evaluation for motorimagery based BCI. J Neural Eng 2013;10:031001.

4. Zhang Q, Hayashibe M, Azevedo-Coste C. Evoked electromyography-based closedloop torque control in functional electrical stimulation. IEEE Trans Biomed Eng 2013; 60(8): 2299-2307.

5. Popovic DB. Advances in functional electrical stimulation (FES). J Electromyography \& Kinesiology 2014;24:795-802.

6. Meng $\mathrm{F}$, Yu Tong $\mathrm{K}$, Tak Chan $\mathrm{S}$, et al. BCI-FES training system design and implementation for rehabilitation of stroke patients. In: IJCNN 2008: Proceedings of the IEEE International Joint Conference on Neural Networks, 2008 Jun 1-8, Hongkong. IEEE; 2008. pp 4103-4106.

7. Yao L, Zhang D, Huang G, Zhu X. Using SSVEP based brain-computer interface to control functional electrical stimulation training system. In: Proceedings of IEEE $5^{\text {th }}$ International Conference on Cybernetics and Intelligent Systems (CIS),
2011 Sept 17-19, Qingdao, China. IEEE; 2011. pp 323-328.

8. Gollee H, Volosyak Y, McLachlan J, et al. An SSVEP-based brain-computer interface for the control of functional electrical stimulation. IEEE Trans Biomed Eng 2010;57:1847-55.

9. Takahashi M, Gouko M, Ito K. Electroencephalogram (EEG) and functional electrical stimulation (FES) system for rehabilitation of stroke patients. In CBMS 2008: Proceedings of IEEE International Symposium on Computer-Based Medical Systems, 2008 June 17-19, Jyvaskyla, Finland. IEEE; 2008. pp 53-58.

10. Sanei S, Chambers J. EEG signal processing. Wiley-Interscience; 2007.

11. Lu H, Plataniotis KN, Venetsanopoulos AN. Regularized common spatial patterns with generic learning for EEG signal classification. In: EMBC 2009: 2009 Annual International Conference of the IEEE Engineering in Medicine and Biology Society, 2009 Sept 3-6, Minneapolis, US. IEEE; 2009. pp 65996602.

12. Alpaydin E. Introduction to Machine Learning (Adaptive Computation and Machine Learning). The MIT Press; 2004.

13. Herman P, Prasad G, McGinnity TM, Coyle D. Comparative analysis of spectral approaches to feature extraction for EEG-based motor imagery classification. IEEE Trans Neural Sys \& Rehab Eng 2008;16:317-26.

14. Bhattacharyya S, Shimoda S, Hayashibe M. A synergetic brain-machine interfacing paradigm for multi-DOF robot control," IEEE Trans Sys, Man, \& Cybernetics: Sys. Accepted for Publication. 